\title{
Verzögertes Abnabeln - Frauen kompetent beraten
}

Anna Kalbér, Thomas Kühn

Wann ist der optimale Zeitpunkt für das Abnabeln Neugeborener - gibt es diesen überhaupt? Was spricht für spätes Abnabeln? Und wie beraten wir Frauen? Der Artikel fasst aktuelle Evidenz zusammen und vermittelt Wissen für die Praxis.

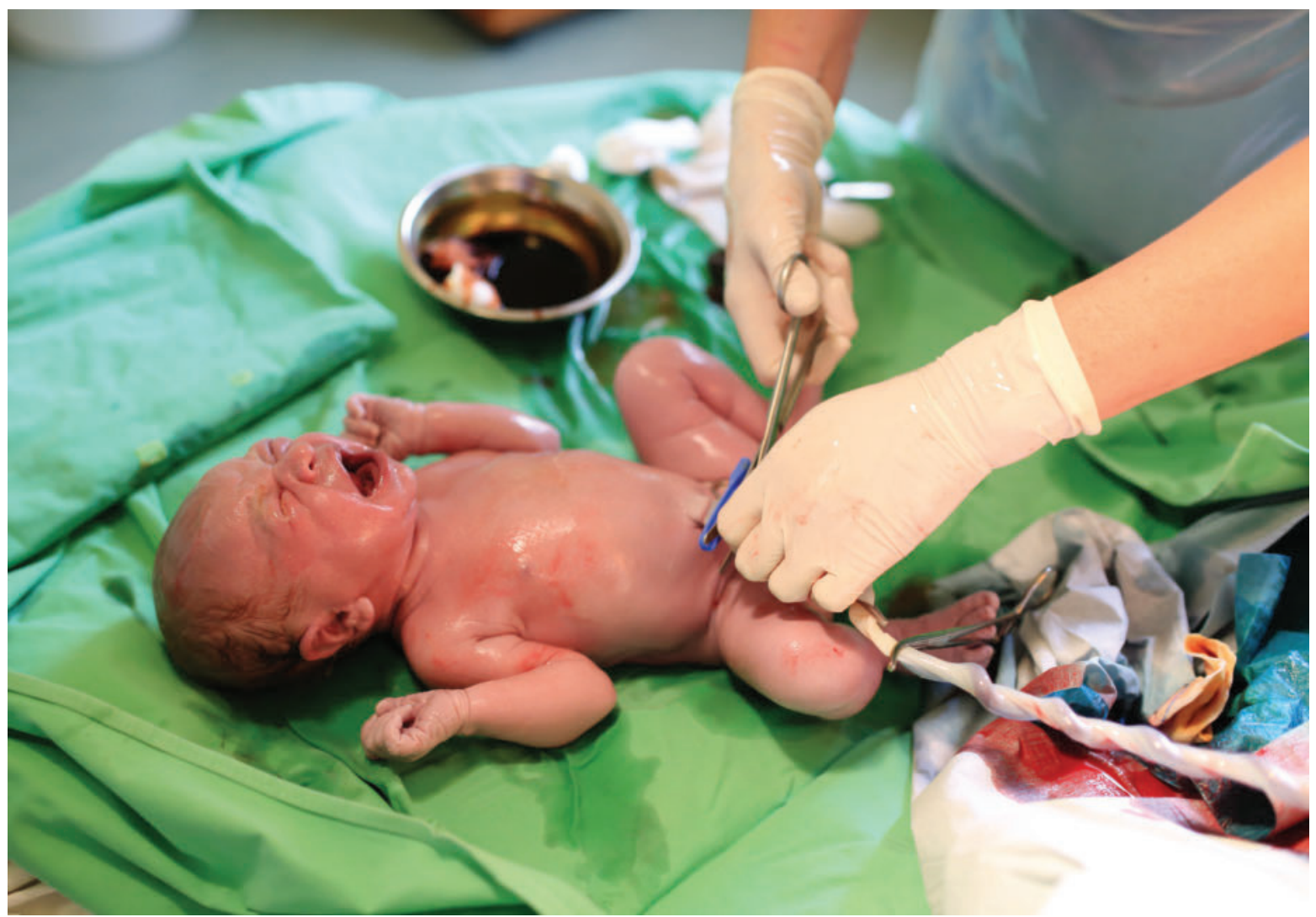

Abb. 1 Der Zeitpunkt für die Abnabelung Neugeborener variiert in geburtshilflichen Einrichtungen erheblich. (Foto: Zsolt Biczó stock.adobe.com, Symbolbild)

\section{Hintergrund}

Unter Abnabelung versteht man im ursprünglichen Sinne das Abtrennen oder Abfallen der Nabelschnur mitsamt der Plazenta des Neugeborenen. Bezogen auf die heutige geburtshilfliche Praxis bezeichnet der Begriff die aseptische Durchtrennung der Nabelschnur nach der Geburt mittels Skalpell oder Schere (Omphalotomie). Dies erfolgt in der Regel relativ einheitlich zwischen zwei angelegten Nabelklemmen. Der am Bauch verbleibende Nabelschnurrest wird gesäubert und vertrocknet innerhalb weniger Tage, bis er schließlich abfällt. Der Bauchnabel bleibt als sichtbarer Teil bestehen.

Dies ist der eigentliche und natürliche Vorgang der Abnabelung. Im übertragenen Sinne wird der Begriff 
Abnabelung für den Ablösungsprozess von Eltern oder anderen Bezugspersonen verwendet [26].

\section{Zeitpunkt der Abnabelung}

In geburtshilflichen Einrichtungen bestehen erhebliche Variationen hinsichtlich des Zeitpunkts der Abnabelung. Hier wird unterschieden zwischen:

- Sofortabnabelung unmittelbar nach der Geburt

- Frühabnabelung etwa 1,5-2 min nach der Geburt

- Spätabnabelung erst nach dem vollständigen Übertritt des Plazentabluts mit dem Erlöschen des Nabelschnurpulses („Auspulsieren“).

Aktuelle Studienansätze wenden sich von einem strikt festgelegten Zeitpunkt des Abnabelns ab. Der Fokus liegt vielmehr auf einem „baby-directed“- oder „physiological-based“ Ansatz zur Abnabelung. Die Durchtrennung der Nabelschnur sollte demnach vorzugsweise erst nach Etablierung einer ausreichenden Belüftung der Lunge erfolgen [9].

\section{STICHWORT}

\section{Lotus-Geburt}

Bei der sog. Lotus-Geburt (englisch: lotus birth) wird die Nabelschnur nach der Geburt möglichst steril und unabgeklemmt mit der entwickelten Plazenta am Neugeborenen belassen, um so die natürliche Abnabelung nach ca. 3-10 Tagen abzuwarten. Beobachtet wurde dieses Verhalten angeblich bereits in den 1960er-Jahren bei Schimpansen. Diese Behauptung stellte sich später jedoch als falsch heraus. Die Methode verbreitet sich seit den 1970er-Jahren vor allem in esoterischen Kreisen. Zur Verhinderung bakterieller Fäulnisvorgänge wird die Plazenta bei der Lotus-Geburt gewaschen, getrocknet und mit Salz und Kräutern in atmungsaktivem Material (Mullwindeln) konserviert. Im Internet sind dafür von privaten Anbietern sog. Lotus-Sets und Plazenta-Taschen erhältlich. Befürworter postulieren, dass durch die Lotus-Geburt die spirituelle Geborgenheit der fetoplazentaren Einheit auch nach der Geburt fortbestehen würde. Dadurch sei eine geringere Traumatisierung und bessere Mutter-Kind-Bindung gegeben. Auch wird, neben zahlreichen anderen gesundheitlichen Vorteilen für das Neugeborene, ein Zuwachs an Nährstoffen, Vitaminen, Mineralien, Eisen und Stammzellen angenommen [15].

Letzteres ist sicherlich nicht der Fall, da mit dem Auspulsieren der Nabelschnur schon nach wenigen Minuten der Blut- und Nährstoffaustausch zwischen Plazenta und Kind vollständig endet. Die vermeintlichen medizinisch-physiologischen Vorteile der Lotus-Geburt sind also ausschließlich Argumente für spätes Abnabeln und nicht für die tagelange Verbindung des Kindes mit der Plazenta. Zudem kann bei nicht sachgemäßer Anwendung dieser Methode ein Infektionsrisiko für das Neugeborene durch aufsteigende Keime bestehen. 2008 führte dies zu einer entsprechenden Warnung durch das britische Royal College of Obstetricians and Gynaecologists [29]. Bei richtiger Anwendung erscheinen die Risiken der Lotus-Geburt allerdings eher gering. Die oft angeführten Vorteile haben jedoch keinerlei wissenschaftliche Evidenz.

\section{Abnabeln nach Termingeburt}

Über den richtigen Zeitpunkt des Abnabelns gibt es immer wieder unterschiedliche Auffassungen und zuweilen auch Kontroversen.

\section{Frühes Abnabeln}

Der britische Arzt Erasmus Darwin erwähnte schon im frühen 19. Jahrhundert: „Eine weitere sehr schädliche Sache für das Kind ist das zu frühe Binden und Schneiden der Nabelschnur, die immer so lange verbleiben sollte, nicht nur bis das Kind wiederholt atmet, sondern bis das Pulsieren der Nabelschnur beendet ist, ansonsten ist das Kind viel schwächer. . ." [11][13]

Frühes Abnabeln (syn.: ECC - Early cord clamping) hat in der Geburtshilfe eine lange Tradition. Oft ist es der stolze Vater, der sofort die Nabelschnur durchtrennen darf. Argumentativ soll eine rasche Abnabelung zuverlässigere Nabelschnurparameter $(\mathrm{NapH} / \mathrm{NvpH})$ gewährleisten und auch frühe postpartale Nachblutungen verhindern. Beide Argumente beruhen auf Fehlannahmen, da der mütterliche Kreislauf nicht direkt mit der Nabelschnurzirkulation verbunden ist und die Blutabnahme zur pH-Bestimmung durchaus auch noch bei verbundener Nabelschnur erfolgen kann. Mehrere Cochrane-Analysen konnten inzwischen zeigen, dass keine erhöhten Risiken für postpartale Nachblutungen, Anämien oder für einen erhöhten Transfusionsbedarf bei den Müttern bestanden [22][26][28].

Wissenschaftlich belegt ist dagegen, dass die Sofortabnabelung eines Neugeborenen unmittelbar nach der Geburt, die kindlichen Eisenvorräte beschneidet. Der Grund dafür ist, dass eine größere Blutmenge in der Nabelschnur zurückbleibt und nicht in den Kreislauf des Kindes gelangen kann.

\section{Warum verzögert abnabeln?}

Bei verzögertem Abnabeln (syn.: DCC - Delayed cord clamping) findet dagegen sozusagen eine autologe Bluttransfusion aus der Plazenta statt: Das Blutvolumen bei 
spät abgenabelten Neugeborenen ist etwa um ein Drittel (ca. $80 \mathrm{ml}$ nach $1 \mathrm{~min}$, ca. $100 \mathrm{ml}$ nach $3 \mathrm{~min}$ ) höher als bei sofort abgenabelten Kindern [19][20][39].

\footnotetext{
Merke

Beim späten Abnabeln sind die kindlichen Eisenspeicher (Ferritin) infolge des bis zu 30-40 \% höheren neonatalen Blutvolumens deutlich aufgefüllt. Damit ist die Eisenversorgung in den ersten Lebensmonaten verbessert und Anämien wird vorgebeugt.
}

So waren die Ferritinspiegel bei Säuglingen im Alter von 4 Monaten in einer schwedischen Studie $(n=121 / 141)$ um $45 \%$ erhöht. Eine Eisenmangelanämie, deren Prävalenz in Europa immerhin bei $2-7 \%$ liegt, konnte so vermieden werden. Im weiteren Verlauf zeigten die spät abgenabelten Kinder bessere feinmotorische Fähigkeiten und eine höhere Sozialkompetenz. Ursächlich dafür könnte eine bessere Myelinisierung der Nervenfasern infolge erhöhter Eisenvorräte sein. Dies erscheint bedeutsam, da ein frühkindlicher Eisenmangel weltweit ein großes Problem darstellt und zudem mit einem schlechteren neurologischen und kognitiven LangzeitOutcome einhergeht [3][4].

\section{Was sagen die Leitlinien?}

Die WHO hat bereits 2014 in einer Richtlinie das verzögerte Abnabeln (im Zeitraum>1-3 min nach der Geburt) empfohlen. Die Richtlinie gilt für Neugeborene und auch für Frühgeborene, die keiner unmittelbaren Reanimation bedürfen. Bei ausreichender Erfahrung im Team sollte im Rahmen der neonatalen Basisreanimation die Nabelschnur sogar erst nach ausreichender Lungenbelüftung durchtrennt werden [37].

Eine weitere Empfehlung ist der aktuell gültigen, jedoch schon länger zur Überarbeitung anstehenden deutschen Leitlinie zur Betreuung von gesunden, reifen Neugeborenen in der Geburtsklinik zu entnehmen. Darin wird empfohlen, bei vaginal geborenen Kindern frühestens nach 1-1,5 min abzunabeln oder ein Auspulsieren der Nabelschnur abzuwarten, da dieses Vorgehen die Erythrozytenzahl und die Eisenspeicher des Neugeborenen erhöht [3][6][36]. Zum Vorgehen nach Sectio werden in der Leitlinie keine evidenzbasierten Aussagen getroffen, allerdings sollte der Geburtsmodus hier eigentlich kein unterschiedliches Vorgehen induzieren.

Die AAP (American Academy of Pediatrics), das ACOG (American College of Obstetricians and Gynaecologists) und das ACNM (American College of Nurse-Midwives) empfehlen ebenso ein um mindestens 30-60 sek verzögertes Abnabeln Reifgeborener. Begründet wird dies durch einen konsekutiv erhöhten Hämoglobinspiegel und Eisenspeicher während der ersten Lebensmonate und die möglicherweise daraus resultierende bessere neurologische Entwicklung [1]. 


\section{Studienergebnisse zum Abnabeln nach Termingeburt}

Das bereits oben zitierte Cochrane-Review untersuchte 2013 den Zeitpunkt des Abnabelns bei Termingeborenen und beurteilte 15 klinische Studien mit 3911 Müttern und deren Einzelkindern. Frühes Abnabeln (< $1 \mathrm{~min}$ ) resultierte in signifikant niedrigeren Hämoglobinspiegeln, sowohl unmittelbar nach der Geburt (gewichteter mittlerer Unterschied -2,17 g/ dL; $95 \%$ confidence interval $\mathrm{Cl},-4,06$ bis $-0,28)$ als auch nach $24-48$ std pp (gewichteter mittlerer Unterschied $-1,49 \mathrm{~g} / \mathrm{dL} ; 95 \% \mathrm{Cl}$, $-1,78$ bis $-1,21$ ) im Vergleich zu den spät abgenabelten Neugeborenen (> 1 min oder nach Auspulsieren).

Gleichzeitig gibt es immer wieder Befürchtungen hinsichtlich einer erythrozytären Überladung des fetalen Kreislaufsystems (Polyzytämie) mit nachfolgender Polyglobulie und prolongierter Hyperbilirubinämie nach verzögertem Abnabeln. In der zitierten Cochrane-Analyse traten Polyzytämien nicht häufiger auf, allerdings war eine etwas höhere Rate an therapiepflichtigen Hyperbilirubinämien (2,74\% ECC-Gruppe versus $4,36 \%$ LCCGruppe; RR, 0,62; $95 \% \mathrm{Cl}, 0,41$ - 0,96) zu beobachten. Hierzu ist die vorliegende Studienlage allerdings noch inkonsistent [22].

Die Langzeiteffekte verzögerten Abnabelns für die neurologische Entwicklung waren bisher nur Gegenstand weniger Studien: Andersson und Mitarbeiter fanden in einer vierjährigen Kohortenstudie nach 4 und 12 Monaten keine Unterschiede im IQ und in der neurologischen Entwicklung der Kinder. Im Alter von 4 Jahren zeigten früh abgenabelte Kinder jedoch moderat niedrigere Scores für Feinmotorik und Sozialverhalten. Ob dies tatsächlich auf die bessere Eisenversorgung kurz nach der Geburt zurückzuführen ist, bleibt zu prüfen [4].

\section{Zeitpunkt des Abnabelns im Kontext sozio-ökonomischer Gegebenheiten}

Der Eisenbedarf eines Säuglings wird in den ersten 6 Monaten gut über ausschließliche Muttermilchernährung gedeckt. Nach diesem Zeitpunkt empfehlen nationale Fachgesellschaften, UNICEF und WHO - auch vor dem Hintergrund einer ausreichenden Eisenzufuhr - den Beginn einer Zufütterung unter fortgesetzter Muttermilchernährung. Unter weiterem ausschließlichen Stillen zeigt sich gerade in Ländern mit schwierigen sozio-ökonomischen Gegebenheiten bei Kindern, die älter als 6 Monate sind, häufig ein latenter oder auch klinisch relevanter Eisenmangel.

Den Effekt eines verzögerten Abnabelns auf die kindliche Entwicklung im ersten Lebensjahr, hat kürzlich eine Forschergruppe in Kathmandu (Nepal), einer
Region mit hoher Prävalenz für Eisenmangelanämien, geprüft: In einer randomisierten-kontrollierten Studie wurden 540 Reifgeborene entweder früh (ECC - Early cord clamping innerhalb von 60 Sekunden) oder in der Interventionsgruppe erst nach 3 min oder mehr (DCC Delayed cord clamping) abgenabelt. Nach 12 Monaten wurden Eltern mit Hilfe des ASQ-Fragebogens (Ages and Stages Questionnaires) interviewt. Für 332 Kinder $(61,5 \%)$ lagen Ergebnisse vor. Eine Einstufung unterhalb einer Standardabweichung des Mittelwerts der Gesamtgruppe wurde als erhöhtes Risiko für eine beeinträchtigte neurologische Entwicklung eingeschätzt. Für 18,1 \% der ECC-Kinder traf dies zu, in der DCC-Interventionsgruppe waren es nur 7,8\%. Das relative Risiko lag damit bei 0,43 (Konfidenzintervall KI 0,26-0,71) zugunsten der spät abgenabelten Kinder. Spät abgenabelte Neugeborene waren am Ende des ersten Lebensjahres den früh abgenabelten Kindern in den Einzelkriterien „Kommunikation“ $(p=0,008)$, „Motorik“ $(p=0,02)$ und „personell-sozial“ $(p=0,008)$ signifikant überlegen [24].

Merke

Eine Abnabelung nach frühestens 3 min stellt ein einfaches, kostengünstiges Verfahren dar, das auch in Lowincome-settings rasch zu etablieren ist, um Hämoglobinstatus, Eisenspeicher und damit die neurologischen Entwicklungschancen im Säuglingsalter zu verbessern.

In vielen Geburtskliniken wird heute verzögert abgenabelt, allerdings ist die Variationsbreite erheblich. Sie reicht von 30 sek über 1, 2 oder sogar 3 min. Andere Kliniken praktizieren vor allem bei thermoinstabilen Frühgeborenen ein mehrmaliges Ausstreichen der Nabelschnur in Richtung Neugeborenem (Cord milking).

\section{Abnabeln von unreifen Frühgeborenen}

Bei Frühgeborenen ist die Studienlage hinsichtlich des Abnabelns noch sehr heterogen. Lang erwartet wurde die aktuelle prospektiv-randomisierte australische Studie „Australian Placental Transfusion Study“ ( $n=1566$, median 28 SSW). Bei Frühgeborenen<30 Schwangerschaftswochen (SSW) mit raschem Abnabeln<10 sek (median 5 sek) erbrachte die Studie in den primären Endpunkten Tod binnen 6 Wochen oder schwere Morbidität durch höhergradige Hirnblutungen, Sepsis, NEC, BPD oder erhebliche Retinopathie keinen signifikanten Unterschied zur Kontrollgruppe mit verzögertem Abnabeln > 1 min (median 60 sek). Allerdings war die Frühmortalität mit $8,9 \%$ bei rascher Abnabelung (70 von 782 Kindern) signifikant höher $(p=0,03)$ als bei verzögert abgenabelten Kindern mit 6,4\% (50 von 784). Nach Meinung der Autoren spricht das Studienergebnis nicht dafür, generell eine verzögerte Abnabelung zu 
empfehlen, wie es einige Fachgesellschaften bereits tun. Hierbei muss allerdings kritisch angemerkt werden, dass selbst diese relativ große Studie keine ausreichende Fallzahl aufwies, um Unterschiede hinsichtlich der primären Endpunkte Tod oder schwere Morbidität nachzuweisen [33].

Andere Studien legen jedoch schon seit Längerem ein verzögertes Abnabeln nahe, gerade auch bei sehr unreifen Frühgeborenen: 2012 fand ein systematisches Studienreview zur Spätabnabelung (in diesem Fall>30 - max. 180 sek) 15 klinische Studien mit 738 Frühgeborenen zwischen 24 und 36 Schwangerschaftswochen [7][28].

\section{Es zeigten sich erhebliche Unterschiede in der Kurzzeit-} morbidität nach plazento-fetaler Transfusion:

- stabilere postnatale Blutzirkulation mit höheren systemischen Blutdrücken und geringerem Bedarf an inotropen Medikationen

- höherer Bluthämatokrit und damit ein verringerter Transfusionsbedarf (7 Studien, 392 Kinder; Relatives Risiko [RR], 0,61; $95 \% \mathrm{Cl} 0,46-0,81$ )

- eine niedrigere Hirnblutungsrate (10 Studien, 539 Kinder; RR 0,59; $95 \%$ Cl, 0,41-0,85)
- geringere NEC-Inzidenz (Nekrotisierende Enterokolitis) (5 Studien, 241 Kinder; RR, 0,62; $95 \%$ Cl, $0,43-0,90)$

Kombiniert man die Ergebnisse der australischen Studie mit den anderen bereits vorliegenden Untersuchungen in einer Meta-Analyse, zeigt sich eine signifikante Verringerung der Mortalität von Frühgeborenen im Krankenhaus, bei denen die Abnabelung verzögert erfolgte [14].

Merke

In den europäischen Konsensus-Guidelines zum

Management des neonatalen Atemnotsyndroms, im

Update 2016 und wiederholt 2019 wird der Zeitpunkt

des Abnabelns als wesentlich herausgestellt [31]. Die

jüngst aktualisierte Fassung empfiehlt daher eine

Abnabelung Frühgeborener frühestens nach $1 \mathrm{~min}$

[32]. Hierbei ist die unbedingte Thermostabilität des

Kindes jedoch eine unverzichtbare Voraussetzung.

Diesbezüglich optimierte und genau durchgeplante räumliche und organisatorische Bedingungen sowie ein erfahrenes neonatologisches Team beherrschen durchaus auch bei Extremfrühgeborenen eine späte Abnabelung. 


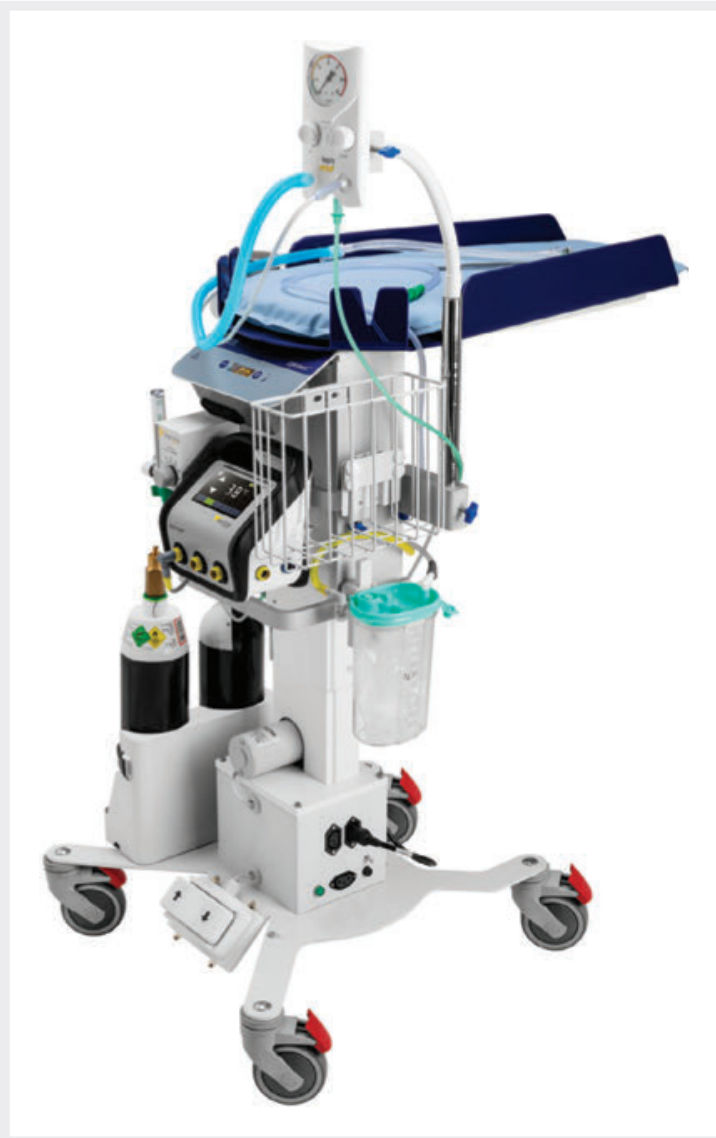

Abb. 2 Technischer Helfer beim späten Abnabeln: Der Reanimationstisch ermöglicht die Erstversorgung des Neugeborenen direkt bei der Mutter. Selbst wenn ein Kind einer sofortigen Intervention bedarf, muss die Nabelschnur nicht sofort durchtrennt werden. (Foto: LifeStart ${ }^{\top \mathrm{M}}$ - Inspiration Healthcare)

Als Nebenwirkung der verzögerten Abnabelung wurden zuweilen höhere Bilirubin-Spitzenspiegel in den LCCGruppen (Late cord clamping) gemessen. Signifikante Unterschiede hinsichtlich der Phototherapie-Behandlungsnotwendigkeit konnten dabei jedoch nicht ermittelt werden. Die Mortalität und die Inzidenz schwerster Hirnblutungen oder periventrikulärer Leukomalazien waren nicht unterschiedlich. Eine Studie zeigte bessere motorische Fähigkeiten spät abgenabelter Frühgeborener $<32$ SSW bei einem korrigierten Alter von 18-22 Monaten [21].

\section{Transmission / Reanimation mit intakter Nabelschnur}

Pathophysiologisch sinnvoll erscheint der in Tiermodellen recht gut evaluierte Ansatz einer respiratorischen Stabilisierung bei noch erhaltener Blutzirkulation über die Plazenta sowohl bei sehr unreifen Frühgeborenen als auch bei adaptationsgestörten Termingeborenen. Diesem liegen folgende Überlegungen zugrunde: Infolge des hohen Gefäßwiderstands fließen bis zur Geburt nur ca. $10 \%$ des rechtsventrikulären kardialen Outputs in die fetalen Lungen. Der Hauptteil des Volumens fließt über die Aorta ascendens und den Ductus arteriosus an der Lunge vorbei. Nach dem Abnabeln und der ersten Lungenbelüftung fällt der pulmonale Gefäßwiderstand rapide $a b$, die zirkulatorische Lungenumgehung reduziert sich rasch und das Blut fließt direkt in das pulmonale Kapillargebiet. Bei einer sofortigen Abnabelung und noch nicht eröffneter Lungenstrombahn führt dies nicht selten zu einer neonatalen Hypoxämie mit konsekutiver Bradykardie infolge der transienten Verminderung des linksventrikulären Outputs. Ein verzögertes Abnabeln bis zur erfolgten postnatalen Lungenbelüftung und der damit stabilisierten Füllung des linken Vorhofs könnte zu einer sanfteren perinatalen Adaptation ohne erhebliche Blutdruckschwankungen führen [8][9].

Dies impliziert jedoch, die initiale neonatologische Erstversorgung direkt bei der spontan oder per Sectio entbundenen Mutter ohne vorherige Durchtrennung der Nabelschnur durchzuführen. Damit wird das oft sehr instabile Frühgeborene weiter via Plazenta oxygeniert, während die initiale Lungenbelüftung zur Etablierung einer funktionellen Residualkapazität (FRC) durch das neonatologische Team schonend und ohne Zeitdruck erfolgen kann.

\section{Studie zur Erstversorgung bei intakter Nabelschnur}

In einer ersten Machbarkeits-Studie untersuchte dies eine Arbeitsgruppe aus den Niederlanden. Die Kriterien zum Abnabeln waren hier folgende [10]:

- adäquat erscheinende Atmung, respektive Lungenbelüftung (mittleres exspiratorisches Atemzugvolumen $\mathrm{Tv} \geq 4 \mathrm{ml} / \mathrm{kg}$ ) unter CPAP-Applikation

- Herzfrequenz $>100$ Schläge / min

- SpO2 > 25. Perzentile bei FiO2 <0,4 [38]

Dabei verwendete die Arbeitsgruppe einen speziell konstruierten Erstversorgungsplatz mit dem Namen "Concord“, der sich in unmittelbarer Nähe der gebärenden Mutter befand. Die Studienpopulation umfasste Frühgeborene mit einem Gestationsalter von 26-35 SSW. Abgenabelt wurde erst, wenn das Kind Zeichen einer erfolgreichen respiratorischen Anpassung nach obigen Kriterien zeigte und angenommen werden konnte, dass eine funktionelle Residualkapazität (FRC) in den Lungen etabliert war. Eingeschlossen wurden 37 Frühgeborene. Die mittlere Zeit bis zum Abklemmen der Nabelschnur betrug 4,23 min (Inter quartal range [IQR] 3,00-5,11). Die Herzfrequenz war nahezu bei allen Kindern stabil. Nur ein Frühgeborenes entwickelte eine Bradykardie $<60$ Schläge/min während der Lagekorrektur der Beatmungsmaske. Allerdings wiesen ca. $50 \%$ der Kinder eine Körpertemperatur von $<36^{\circ} \mathrm{C}$ bei Aufnahme auf 
der NICU auf, was auf einen deutlichen Optimierungsbedarf hinweist.

Erforderlich für ein solches Vorgehen sind damit speziell konstruierte Erstversorgungsplätze, die eine Stabilisierung des Kindes direkt neben der Mutter ermöglichen und dabei kein Risiko hinsichtlich einer neonatalen Hypothermie entstehen lassen. Besonders anspruchsvoll wird dieses Vorgehen unter den sterilen Kautelen eines Kaiserschnitts unter OP-Bedingungen [12][34].

Dieser neu gewählte physiologischere Ansatz zum Cord clamping erscheint bedeutsam, zeigt er doch deutlich:

- dass das derzeit häufig favorisierte Abnabeln nach 1 min tatsächlich nur ein Viertel der Zeit bedeutet, die eine Frühgeborenenlunge zur Entfaltung und FRCEtablierung benötigt

- dass ein verzögertes Abnabeln oder gar Ausstreichen der Nabelschnur bei nicht oder nicht suffizient atmenden Frühgeborenen allein der Komplexität der postnatalen Adaptation von Lunge und Kreislauf eher nicht gerecht werden kann

- dass es sicher richtig und notwendig ist, der sich eröffnenden Lunge ausreichendes zirkulatorisches Volumen bereitzustellen, jedoch muss sie dann auch ausreichenden Support erhalten (z. B. durch frühe und ausreichend hohe CPAP-Atemunterstützung).

Weitere Studien in dieser Richtung sind damit dringend erforderlich.

\section{Abnabeln vor Reanimation bei neonataler Azidose}

Überträgt man den interessanten physiologischen Ansatz auf die Situation bei der Reanimation schwer asphyktischer Neugeborener, könnte dies zu einer Veränderung des bisherigen Vorgehens führen: Neugeborene mit schwerer Atemdepression und Bradykardie oder Pulslosigkeit, die einer sofortigen Reanimation bedürfen, werden bislang sofort abgenabelt und dem neonatologischen Intensivteam übergeben. Bei diesem Vorgehen verbleiben etwa $20-40 \%$ des fetal-plazentaren Blutes in der Plazenta und stehen dem Neugeborenen daher nicht zur Verfügung.
Hypovolämie und Hypotension sind häufig wichtige Faktoren, die den Reanimationserfolg in Frage stellen. Eine stabilere Hämodynamik könnte die kardiale und zerebrale Perfusion verbessern und so die gefürchteten Folgen hinsichtlich Mortalität und schwerer Langzeitmorbidität (u. a. hypoxisch-ischämische Enzephalopathie, Leukomalazie) reduzieren. Eine Reanimation ohne sofortige Abnabelung am Bett der Mutter, stellt derzeit noch eine große technische Herausforderung dar und dürfte nur in den wenigsten Einrichtungen praktiziert werden. In diesem Dilemma wird häufig versucht, dem Neugeborenen dringend benötigtes Plazentablut durch mehrmaliges Ausstreichen der Nabelschnur in Richtung Kind zuzuführen.

\section{Ausstreichen der Nabelschnur (Cord milking)}

Ein solches 3- bis 5-maliges Cord milking benötigt weniger als 15 sek Zeit und verzögert die Reanimation damit nur unerheblich. Bei Reifgeborenen mit schwerer Azidose wurde kürzlich in einer retrospektiven Analyse untersucht, wie sich ein vorheriges Ausstreichen der Nabelschnur im Vergleich zur sofortigen Abnabelung vor der Reanimation auf das Outcome auswirkt.

Eingeschlossen wurden 157 Reifgeborene mit einem $\mathrm{pH}$-Wert $\leq 7,1$ oder einem Basendefizit > $-12 \mathrm{im}$ venösen Blut oder Nabelschnurblut. Fast alle waren atonisch (1 min Apgar 0 oder 1). Bei 36 Kindern wurde die Nabelschnur ausgestrichen. Diese Gruppe unterschied sich weder in maternalen noch peripartalen Parametern von den Kindern, die sofort nach Abnabelung reanimiert wurden. Hinsichtlich des neonatalen Outcomes (Apgar 1 und $5 \mathrm{~min}$, therapeutische Hypothermie, Hirnschädigung im MRT) ergaben sich keine signifikanten Unterschiede zwischen beiden Gruppen. Doch nur 38\% der Kinder in der Cordmilking-Gruppe benötigten Wiederbelebungsmaßnahmen versus $56 \%$ in der Kontrollgruppe. Eine längere respiratorische Unterstützung benötigten 19\% in der Cord-milking-Gruppe versus $31 \%$ in der Kontrollgruppe. Dieser Unterschied war zwar nicht signifikant, er spricht jedoch dafür, dass atemgestörte und azidotische Neugeborene nach raschem Ausstreichen der Nabelschnur seltener Reanimationsmaßnahmen und / oder einer Beatmung bedürfen [17][37]. 
Anzumerken bleibt allerdings, dass ein prolongiertes Abnabeln (DCC) nicht mit mehrmaligem Ausstreichen der Nabelschnur gleichzusetzen ist. Bei Letzterem erfolgen vielmehr Bolus-Expressionen des in der Nabelschnur vorhandenen Blutes in Richtung Kind.

Zahlreiche vorliegende Studien sind sehr heterogen, was den Zeitpunkt, die Patientengruppe, die Zahl der Ausstreichmanöver und die Kombination mit dem Abnabelungszeitpunkt (ECC oder DCC) betrifft. Follow-up-Daten liegen nicht vor und es bestehen Zweifel bezüglich der Sicherheit, vor allem bei sehr unreifen Frühgeborenen: In Tierversuchen zeigten sich ernstzunehmende hämodynamische Veränderungen durch ein Ausstreichen der Nabelschnur. Eine kürzlich durchgeführte klinische Studie hat gezeigt, dass sich das Risiko einer schweren intraventrikulären Blutung durch Cord milking verglichen mit einer verzögerten Nabelschnurklemmung bei Frühgeborenen vervierfacht. Die Sicherheit dieses Verfahrens muss daher in Frage gestellt werden [18].

Eine Meta-Analyse vorliegender Studien ( $n=255$; $G A<33$ SSW) fand zunächst ähnliche Vorteile wie bei verzögertem Abnabeln hinsichtlich der Vermeidung von Hirnblutungen und erhöhten Hämoglobin- und Ferritinkonzentrationen, ohne jedoch schwerwiegende Nebenwirkungen zu verifizieren [23]. Eine Folgestudie ergab bessere kognitive und sprachliche Ergebnisse bei denjenigen Frühgeborenen, die für das Nabelschnur-Ausstreichen randomisiert wurden [1][2][18][25][30]. Derzeit laufen mehrere Studien, um mögliche Vorteile und Risiken eines aktiven Nabelschurausstreichens insbesondere bei extremen Frühgeborenen zu evaluieren.

\section{Merke \\ Gegenwärtig gibt es keine ausreichende Evidenz, um das Cord milking, insbesondere bei sehr unreifen Früh- geborenen, eindeutig zu unterstützen oder zu widerlegen.}

\section{FAZIT}

Das verzögerte Abnabeln Neugeborener ist eine einfach zu etablierende, kostenneutrale und evidenzbasierte Maßnahme mit zahlreichen gesundheitlichen Vorteilen für das Kind. Die häufig noch geübte Praxis des sofortigen Abnabelns von sowohl Früh- als auch Termingeborenen sollte verlassen werden: Die Datenlage und auch die internationalen Empfehlungen sprechen eindeutig dafür, Kinder mindestens um 1 min, besser länger verzögert abzunabeln. Abzuwarten bleibt, wie sich die in naher Zukunft zu erwartende, aktualisierte deutsche Leitlinie zur Betreuung des gesunden reifen Neugeborenen in der Geburtsklinik (024-005) hierzu positionieren wird.
Autorinnen / Autoren

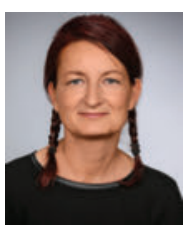

Anna Kalbér ist Kinderkrankenschwester, Case-Managerin und Neotrainer.

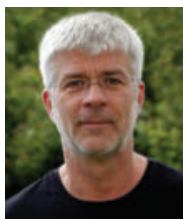

Thomas Kühn ist Kinderarzt, Neonatologe, Stillberater und Neotrainer. Er arbeitet als Oberarzt am Vivantes Perinatalzentrum in Berlin-Neukölln.

\section{Korrespondenzadresse}

E-Mail: info@neotrainer.de

Literatur

[1] American College of Obstetricians and Gynecologist (ACOG). ACOG Guideline, Committee Opinion Number 684: Delayed Umbilical Cord Clamping After Birth. Im Internet: https://www.acog.org/Clinical-Guidance-and-Pub lications/Committee- Opinions / Committee-on-Obstetric- $\mathrm{Pr}$ actice/Delayed-Umbilical-Cord-Clamping-After-Birth. Stand: Januar 2017

[2] Al-Wassia H, Shah PS. Efficacy and safety of umbilical cord milking at birth: a systematic review and meta-analysis. JAMA Pediatr 2015; 169 (1): 18-25

[3] Andersson O, Hellström-Westas L, Andersson D et al. Effect of delayed versus early umbilical cord clamping on neonatal outcomes and iron status at 4 months: a randomized controlled trial. BMJ 2011; 343

[4] Andersson O, Domellöf M, Andersson D et al. Effect of delayed vs early umbilical cord clamping on iron status and neurodevelopment at age 12 months: a randomized clinical trial. JAMA Pediatr 2014; 168: 547-554

[5] Andersson O, Lindquist B, Lindgren $\mathrm{M}$ et al. Effect of Delayed Cord Clamping on Neurodevelopment at 4 Years of Age: A Randomized Clinical Trial. JAMA Pediatr 2015; 169 (7): 631638

[6] AWMF Leitlinie. Betreuung des gesunden reifen Neugeborenen in der Geburtsklinik. 2012. Registernummer 024-005

[7] Berger R, Söder S, Abele $\mathrm{H}$ et al. Neuroprotektion bei Frühgeborenen. Der Gynäkologe 2014; 11: 856-864

[8] Bhatt S, Alison B], Wallace EM et al. Delaying cord clamping until ventilation onset improves cardiovascular function at birth in preterm lambs. J Physiol 2013; 591: 2113-2126

[9] Blank DA, Polglase GR, Kluckow M et al. Haemodynamic effects of umbilical cord milking in premature sheep during the neonatal transition. Archives of Disease in Childhood Fetal and Neonatal Edition 2018; 103: F539-F546

[10] Brouwer E, Knol R, Vernooij ASN et al. Physiological-based cord clamping in preterm infants using a new purpose-built resuscitation table: a feasibility study. Arch Dis Child Fetal Neonatal Ed. Epub ahead of print: [03.10.2018]. doi:10.1136/ archdischild-2018-315483 
[11] Darwin E: The Project Gutenberg ebook of zoonomia. Vol. I. Zoonomia, or the laws of organic life (18.06.2014). Im Internet: http://www.gutenberg.org/files/15707/15707-h/15707-h.htm

[12] Duley L, Dorling J, Pushpa-Rajah A et al. Cord Pilot Trial Collaborative Group. Randomised trial of cord clamping and initial stabilisation at very preterm birth. Arch Dis Child Fetal Neonatal Ed. 2018; 103(1): F6-14

[13] Dunn PM. Dr Erasmus Darwin (1731-1802) of Lichfield and placental respiration. Arch Dis Child Fetal Neonatal Ed. 2003; 88: F346-F348

[14] Fogarty M, Osborn DA, Askie L et al. Delayed vs early umbilical cord clamping for preterm infants: a systematic review and meta-analysis. Am J Obstet Gynecol. 2018; 218 (1): 1-18

[15] Dal Pian D. Lotus-Geburt. Frauenheilkunde aktuell 2007; 2: 35-36. Im Internet: http://www.frauenheilkunde-aktuell.ch/ de/fachmagazin/ausgaben/2007-02/frauenheilkunde-aktuell2007-02.pdf

[16] Katheria A, Mercer J, Brown M et al. Umbilical cord milking at birth for term newborns with acidosis: neonatal outcomes. Journal of Perinatology 2017; doi:10.1038/s41372-017-0011-9

[17] Katheria A, Garey D, Truong G et al. A randomized clinical trial of umbilical cord milking vs delayed cord clamping in preterm infants: neurodevelopmental outcomes at 22-26 months of corrected age. J Pediatr. 2018; 194: 76-80

[18] Katheria AC, Reister F, Hummler $\mathrm{H}$ et al. Premature Infants Receiving Cord Milking or Delayed Cord Clamping: A Randomized Controlled Non-inferiority Trial (abstract LB 1). Am J Obstet Gynecol 2019

[19] Linderkamp O. Blood rheology in the newborn infant. Baillieres Clin Haematol 1987; 1: 801-825

[20] Linderkamp O, Nelle M, Kraus $M$ et al. The effect of early and late cord-clamping on blood viscosity and other hemorheological parameters in full-term neonates. Acta Paediatr 1992; 81: 745-750

[21] Mercer JS, Erickson-Owen DA, Vohr BR et al. Effects of placental transfusion on neonatal and 18 month outcomes in preterm infants: a randomized controlled trial. J Pediatr 2016; 168

[22] McDonald S], Middleton P, Dowswell T et al. Effect of timing of umbilical cord clamping of term infants on maternal and neonatal outcomes. Cochrane Database of Systematic Reviews 2013 (7)

[23] Nagano N, Saito M, Sugiura T et al. Benefits of umbilical cord milking versus delayed cord clamping on neonatal outcomes in preterm infants: A systematic review and meta-analysis. PLoS One 2018; 13(8)

[24] Rana N, Kc A, Målqvist M et al. Effect of delayed Cord Clamping of Term babies on neurodevelopment at 12 Months: $\mathrm{A}$ Randomized Controlled Trial. Neonatology 2018; 115: 36-42

[25] Patel S, Clark EAS, Rodriguez CE et al. Effect of umbilical cord milking on morbidity and survival in extremely low gestational age neonates. Am J Obstet Gynecol. 2014; 211 (5): 519. e1-519.e7

[26] Pschyrembel Wörterbuch Sexualität: Stichwort „Abnabelung“. Berlin: Walter de Gruyter; 2006: 1
[27] Rabe H, Reynolds G, Diaz-Rossello J et al. Early versus delayed umbilical cord clamping in preterm infants. Cochrane Database of Systematic Reviews 2004 (4)

[28] Rabe H, Diaz-Rossello JL, Duley L et al. Effect of timing of umbilical cord clamping and other strategies to influence placental transfusion at preterm birth on maternal and infant outcomes. Cochrane Database of Systematic Reviews 2012 (8)

[29] Royal College of Obstetricians and Gynaecologists (2008). RCOG statement on umbilical non-severance or "lotus birth"

[30] Safarulla A. A review of benefits of cord milking over delayed cord clamping in the preterm infant and future directions of research. J Matern Fetal Neonatal Med. 2017; 30 (24): 29662973

[31] Sweet GS et al. European Consensus Guidelines on the Management of Respiratory Distress Syndrome 2016. Update Neonatology 2017; 111: 107-125

[32] Sweet GS, Carnielli V, Greisen G et al. European Consensus Guidelines on the Management of Respiratory Distress Syndrome 2019; Update Neonatology 2019; 115: 432-450

[33] Tarnow-Mordi W, Morris J, Kirby A et al. Delayed versus immediate cord clamping in preterm infants. $\mathrm{N}$ Engl J Med 2017; 377: 2445-2455

[34] Thomas MR, Yoxall CW, Weeks AD et al. Providing newborn resuscitation at the mother's bedside: assessing the safety, usability and acceptability of a mobile trolley. BMC Pediatrics 2014; 14: 135

[35] Tricarico A, Bianco V, Di Biase AR et al. Lotus Birth Associated With Idiopathic Neonatal Hepatitis Pediatrics \& Neonatology 2017; 58 (3): 281-282

[36] van Rheenen P, Brabin BJ. Late umbilical cord-clamping as an intervention for reducing iron deficiency anaemia in term infants in developing and industrialised countries: a systematic review. Ann Trop Paediatr 2004; 24 (1): 3-16

[37] World Health Organization. WHO Guideline: Delayed umbilical cord clamping for improved maternal and infant health and nutrition outcomes. Im Internet: https://www. who.int/ nutrition / publications/guidelines/cord_clamping/ en/; Stand: 2014

[38] White LN, Thio M, Owen LS et al. Achievement of saturation targets in preterm infants $<32$ weeks gestational age in the delivery room Archives of Disease in Childhood. ADC Fetal and Neonatal Edition 2017; 102: F423-F427

[39] Yao AC, Lind J. Effect of early and late cord clamping on the systolic time intervals of the newborn infant. Acta Paediatr Scand 1977; 66: 489-493

\section{Bibliografie}

DOI https://doi.org/10.1055/a-0953-4640

Die Hebamme 2019; 32: 23-31

(C) Georg Thieme Verlag KG Stuttgart · New York ISSN 0932-8122 